\title{
Effects of diphyllin as a novel V-ATPase inhibitor on TE-1 and ECA-109 cells
}

\author{
HAIJIAO CHEN ${ }^{1,2^{*}}$, PENGFEI LIU ${ }^{1,3^{*}}$, TING ZHANG ${ }^{1,2}$, YI GAO $^{1,3}$, YINGDI ZHANG ${ }^{1,3}$, \\ XIUYUN SHEN $^{1,3}$, XIAO LI $^{1,3}$ and WEIDONG SHEN ${ }^{1,3}$ \\ ${ }^{1}$ Jiangsu Key Laboratory of New Drug Research and Clinical Pharmacy, Xuzhou Medical University, \\ Xuzhou, Jiangsu 221000; Departments of ${ }^{2}$ Center Laboratory and ${ }^{3}$ Gastroenterology, \\ The Affiliated Jiangyin Hospital of Nantong University, Wuxi, Jiangsu 214400, P.R. China
}

Received March 7, 2017; Accepted September 1, 2017

DOI: 10.3892/or.2018.6191

\begin{abstract}
Diphyllin is a natural component of traditional Chinese medicine, which effectively inhibits V-ATPase activity and affects the progression of cancer. However, few studies have been conducted on esophageal cancer, and the mechanisms remain to be elucidated. The present study revealedthat diphyllin inhibited proliferation and induced $\mathrm{S}$ arrest in esophageal cancer cell lines TE-1 and ECA-109. Further experiments revealed that diphyllin inhibited V-ATPase activity and decreased the mRNA expression of mammalian target of rapamycin complex 1 (mTORC1), hypoxia-inducible factor- $\alpha$ (HIF-1 $\alpha$ ), and vascular endothelial growth factor (VEGF). The present study also revealed that diphyllin inhibited proliferation and reduced the formation of new blood vessels. Diphyllin inhibited blood metastasis by regulating the mTORC1/HIF-1 $\alpha$-/VEGF pathway, therefore it could be considered as a new V-ATPase inhibitor to treat esophageal cancer.
\end{abstract}

\section{Introduction}

Esophageal carcinoma is a common malignant solid tumor of the digestive tract. An acidic extracellular microenvironment is an important characteristic of solid tumors (1), and acidification of the extracellular environment (even mild acidification) influences cell signaling pathways that affect apoptosis and proliferation. Recent studies revealed that extracellular acidification and the expression of various ion exchange channels in tumor cells were associated with tumor

Correspondence to: Dr Weidong Shen, Department of Gastroenterology, The Affiliated Jiangyin Hospital of Nantong University, Shoushan Road 163, Wuxi, Jiangsu 214400, P.R. China

E-mail: shenwd1976@126.com

${ }^{*}$ Co-first authorship

Key words: diphyllin, V-ATPase, gastric cancer, signaling cell hypoxia and played an important role in the progression of tumors.

Our previous studies and other related studies have indicated that V-ATPase is a proton pump that is closely related to establishment and maintenance of an acidic microenvironment, and is expressed highly in tumor tissues $(2,3)$. Diphyllin is a natural component of traditional Chinese medicine, which belongs to the lignan compounds and has good antitumor activity with relatively few side-effects. However, the exact mechanism is not yet clear. In a previous study, we found inhibition of V-ATPase expression in the human gastric cancer cell line SGC7901 by diphyllin (3). In the present study, we investigated whether diphyllin inhibits V-ATPase activity in esophageal cancer and further explored its role in malignant behavior.

Metastasis of esophageal cancer is the main reason for poor prognoses, and blood circulation is the major pathway of metastasis (4). Vascular endothelial growth factors (VEGFs), including VEGF-A, VEGF-C and VEGF-D, play an important role in the formation of new blood vessels and are related to the mechanism $(5,6)$. Hypoxia-inducible factor- $1 \alpha(\mathrm{HIF}-1 \alpha)$ is an upstream gene of VEGF, which regulates the formation of new vessels. Our previous study revealed that HIF-1 $\alpha$ expression in esophageal cancer was correlated with VEGF$\mathrm{C} /$-D expression and was closely related to the metastasis of esophageal cancer $(7,8)$. In addition, mammalian target of rapamycin (mTOR) is an important regulator of the upstream molecules of HIF-1 $\alpha(9,10)$. mTOR forms two complexes, namely mTORC1 and mTORC2, which play important roles by affecting different signaling pathways. Among them, mTORC1 regulates HIF-1 $\alpha$ (11). Therefore, mTORC1/HIF-1 $\alpha /$ VEGF signaling is a major regulatory signal in the metastasis of cancer.

Previous studies have indicated a significantly lower $\mathrm{pH}$ in lysosomes of tumor cells in vitro than in normal cells, which is conducive to increase autophagy and intracellular free amino acids, thus activating mTORC1 signaling. Furthermore, mature lysosomes and extracellular acidic maintenance are closely related to the role of V-ATPase. Therefore, we hypothesized that diphyllin may inhibit the activity of V-ATPase and mTORC1/HIF-1 $\alpha /$ VEGF signaling. In the present study, we tested our hypothesis and explored the possible mechanism of diphyllin in the inhibition of esophageal cancer metastasis. 


\section{Materials and methods}

Drugs. Diphyllin was kindly provided by Dr Zhaoyu (Nantong University, Jiangsu, China). Diphyllin and bafilomycin A1 (Sigma, St. Louis, MO, USA) were dissolved in dimethyl sulfoxide (DMSO; Sigma) and stored at $-20^{\circ} \mathrm{C}$.

Cell lines and culture. Human esophageal squamous cancer cell lines TE-1 and ECA-109 purchased from the American Type Culture Collection (ATCC; Manassas, VA, USA) were maintained in Dulbecco's modified Eagle's medium (DMEM; HyClone, Logan, UT, USA) supplemented with $10 \%$ fetal bovine serum (FBS; ScienCell, Carlsbad, CA, USA). Reagents for cell culture were purchased from Gibco (Grand Island, NY, USA). The cells were incubated at $37^{\circ} \mathrm{C}$ with $5 \% \mathrm{CO}_{2}$.

In vitro cytotoxicity assay of diphyllin. TE-1 and ECA-109 cells were seeded into a 96-well clear polystyrene microplate (Corning, Corning, NY, USA USA) at 4,000 cells/well at $20 \mathrm{~h}$ prior to the experiment. Diphyllin was 2-fold serially diluted in culture medium and added to the cell monolayer in triplicate. The final DMSO concentration was no $>0.5 \%$ in all wells. After 2 days, the culture supernatant was removed and $100 \mu \mathrm{l}$ DMEM with $10 \mu \mathrm{l}$ Cell Counting Kit-8 (CCK-8) reagent was added to each well, followed by incubation at $37^{\circ} \mathrm{C}$ for $4 \mathrm{~h}$. The absorbance values were assessed at $430 \mathrm{~nm}$ with a reference wavelength of $490 \mathrm{~nm}$ on a Bio-Rad iMark (Bio-Rad, Hercules, CA, USA). Control cells (without diphyllin/bafilomycin A1 treatment) were assumed to represent $100 \%$ cell viability. Normalized cell viability data were plotted against diphyllin concentrations and fitted to a non-linear regression curve using GraphPad Prism (GraphPad Software, San Diego, CA, USA). The $50 \%$ cytotoxicity concentration $\left(\mathrm{IC}_{50}\right.$; the concentration of diphyllin at which cellular viability was reduced to $50 \%$ ) was accordingly obtained.

Enzyme-linked immunosorbent assay (ELISA). TE-1 and ECA-109 cells were grown into a 6-well clear polystyrene microplate with culture medium at $\mathrm{pH}$ 6.6. The levels of VEGF-C in the culture supernatants of TE-1/ECA-109 cells were assessed using a human ELISA kit (Cloud-Clone Corp., Houston, TX, USA) after treatments with diphyllin/bafilomycin $\mathrm{A} 1$ for $6,12,24$ and $48 \mathrm{~h}$.

Colony formation assay. Cells (400/well) were seeded into 6-well plates with $2 \mathrm{ml}$ DMEM containing 10\% FBS in the presence or absence of various concentrations of diphyllin at $37^{\circ} \mathrm{C}$. After culture for 11 days, colonies with cell numbers of $>50$ were counted under a microscope.

Cell cycle detection. Cell cycle detection was performed using a propidium iodide (PI) staining detection kit (KeyGen Biotech, Nanjing, China) by MoFlo XDP (Beckman Coulter, Inc., Brea, CA, USA). Briefly, the cells were trypsinized, washed with phosphate-buffered saline (PBS), centrifuged and fixed with $70 \%$ cold ethanol $(500 \mu \mathrm{l})$ for $2 \mathrm{~h}$, and then washed with PBS. The cells were incubated with $100 \mu 1$ RNase A solution for $30 \mathrm{~min}$ at $37^{\circ} \mathrm{C}$. PI $(400 \mu \mathrm{l})$ was added and the cells were incubated for another $30 \mathrm{~min}$ at $4^{\circ} \mathrm{C}$. The samples were analyzed within $1 \mathrm{~h}$ by MoFlo XDP with Summit software. The data were analyzed by ModFit LT (4.1).

Transwell and scratch wound assays. Transwell chambers (Corning cat. no. 34221; 6.5-mm Transwell with an 8.0- $\mu \mathrm{m}$ pore polycarbonate membrane insert) were placed in 24-well plates (Corning), and 1x10 ${ }^{4}$ ECA-109 cells were cultured/well. Diphyllin/bafilomycin A1 were added after $20 \mathrm{~h}$, and then cell migration was observed after $48 \mathrm{~h}$ of treatment.

TE-1 and ECA-109 cells were grown into 6-well plates (Corning) at $4 \times 10^{5}$ cells/well $20 \mathrm{~h}$ prior to experiments. A scratch wound was made along the bottom of the well with a pipette tip, the scratch area, and then the cells were washed with PBS. After adding fresh medium, cell migration was detected at 12, 24 and $48 \mathrm{~h}$.

V-ATPase activity assay. We employed a colorimetric assay to assess V-ATPase activity using a spectrophotometer to detect the release of phosphate, according to the manufacturer's instructions (V-ATPase assay kit; Genmed Scientifics Inc., Arlington, MA, USA). Briefly, the cells were cultured in complete DMEM at $37^{\circ} \mathrm{C}$ with $5 \% \mathrm{CO}_{2}$. With different solubility of drug-treated cells, the cells were cultured for $48 \mathrm{~h}$ and then collected. The activity of V-ATPase was determined in a quartz cuvette. The sample absorbance at $340 \mathrm{~nm}$ was detected at 0 and $20 \mathrm{~min}$. The experiment was repeated three times.

Real-time quantitative PCR. Total RNA was isolated from ECA-109 cells using TRIzol reagent (Invitrogen, Carlsbad, CA, USA) according to the manufacturer's instructions. Reverse transcription reactions were conducted with a Transcriptor First Strand cDNA Synthesis kit (Takara, Otsu, Japan). PCR primers were designed by BGI (China). Real-time PCR with SYBR-Green PCR Master Mix (Takara) was performed using a CFX96 (Bio-Rad). Fluorescence signals were assessed during the extension phase. Cq values of the sample were calculated, and transcript levels were calculated using the $2^{-\Delta \Delta \mathrm{Cq}}$ method.

Detection of proliferation, migration and tube formation of human umbilical vein endothelial cells (HUVECs) cultured in various culture supernatants. Cells were seeded in 12-well culture plates at $2 \times 10^{6}$ cells/well. After treatment with diphyllin or bafilomycin for 12, 24 and $48 \mathrm{~h}$ culture supernatants were collected, centrifuged and stored at $-80^{\circ} \mathrm{C}$. HUVECs were seeded in 96-well culture plates at $8 \times 10^{3}$ cells/well in $100 \mu \mathrm{l}$ of culture supernatant. Cell proliferation and migration were detected as described above. HUVECs were seeded in 12-well culture plates at $1 \times 10^{5}$ cells/well. After $6 \mathrm{~h}$ of culture, migration and the formation of tube-like structures were observed under a microscope, and the number of lumens was statistically calculated.

Statistical analysis. Statistical comparisons were performed using SPSS 16.0 software package (SPSS, Inc., Chicago, IL, USA), using the Student's t-test for paired observations or one-way analysis of variance with Student-Newman-Keuls, for least significant difference, and Dunnett's tests. All data are presented as the mean \pm standard deviation (SD). $\mathrm{P}<0.05$ was considered as statistically significant $(\mathrm{P}<0.05, \mathrm{P}<0.01)$. Mean 
A

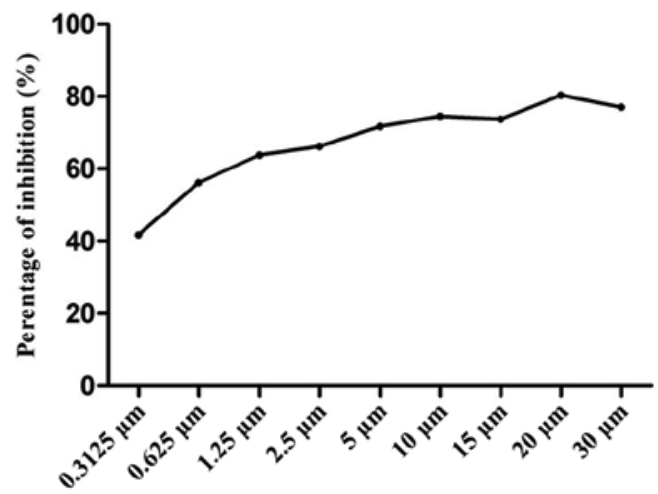

C

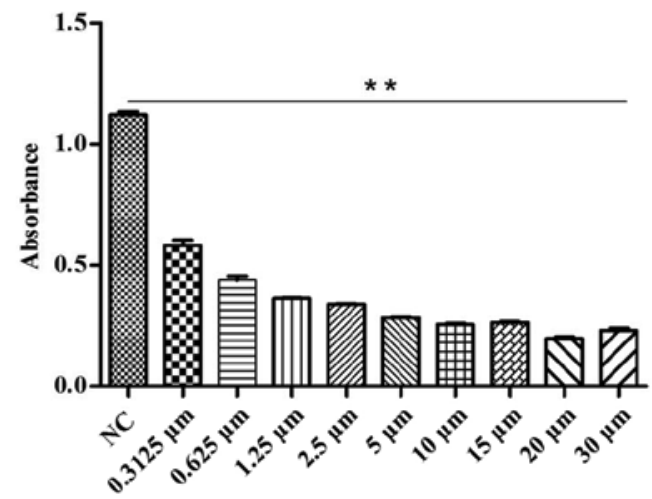

E

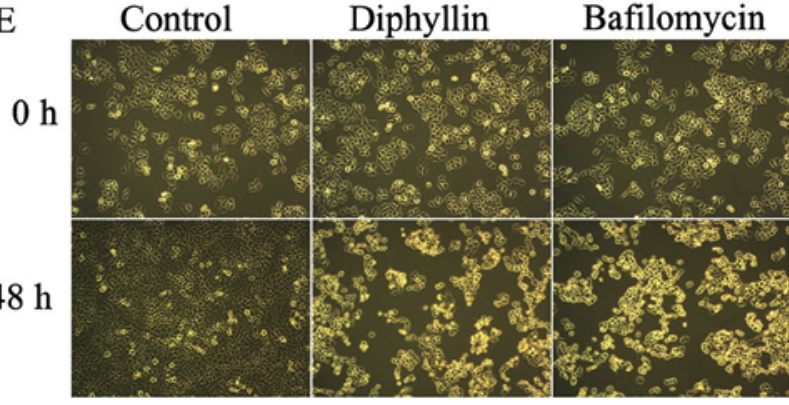

G

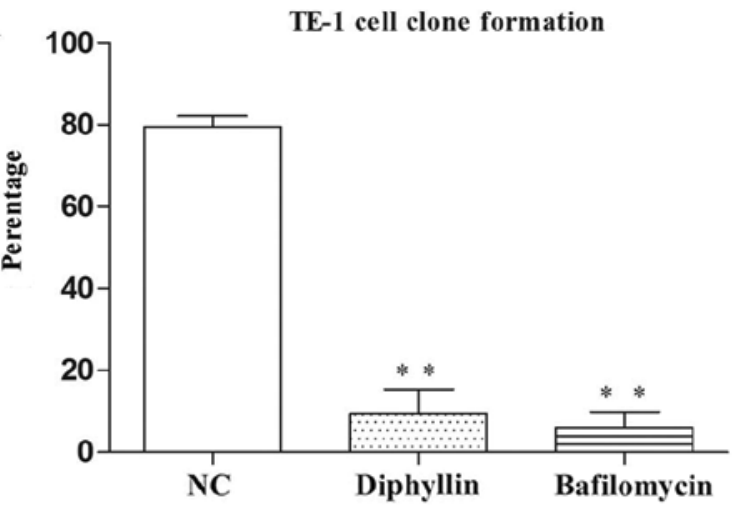

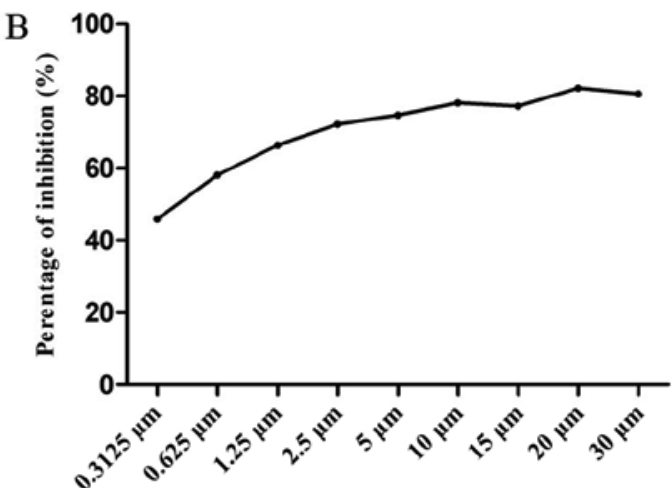

D
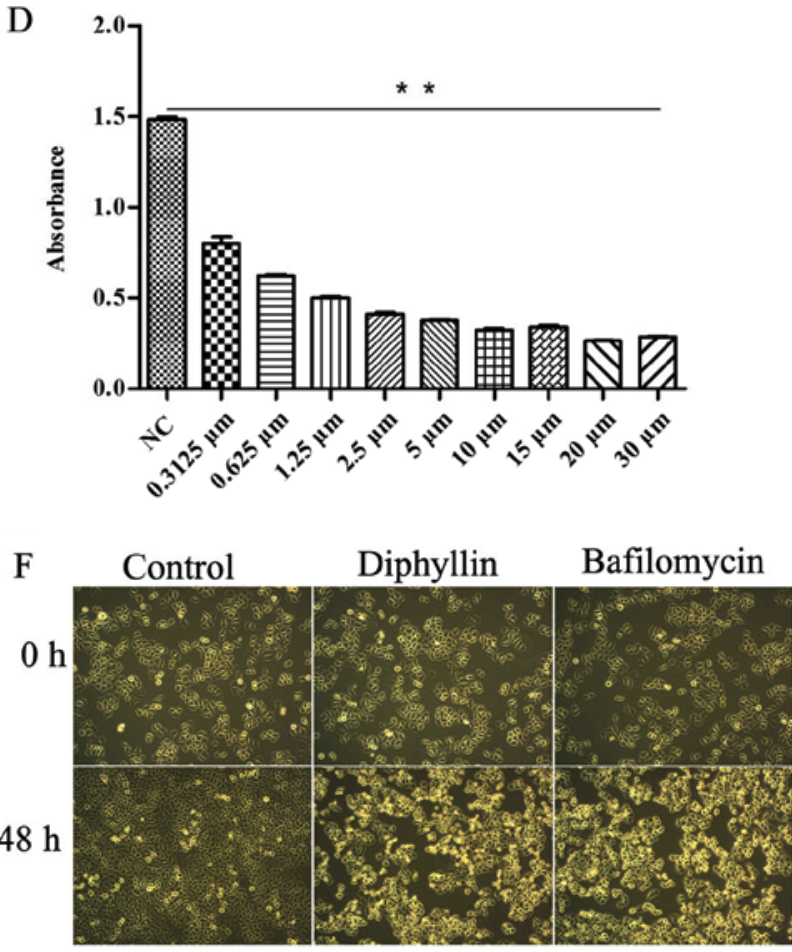

$\mathrm{H}$

ECA-109 cell clone formation

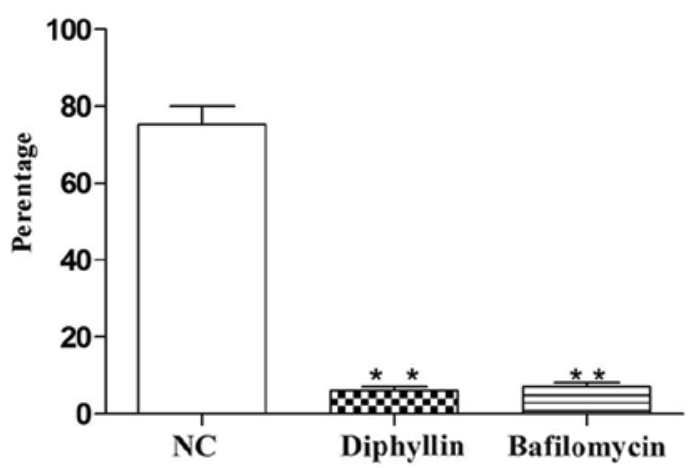

Figure 1. (A and B) $\mathrm{IC}_{50}$ value of TE-1 and ECA-109 cells. (C-F) Inhibition of TE-1 and ECA-109 cell proliferation by diphyllin (magnification, $\mathrm{x} 40$ ). $(\mathrm{G}$ and $\mathrm{H})$ Diphyllin suppressed colony formation of TE-1 and ECA-109 cells; ${ }^{* *} \mathrm{P}<0.01$.

values and SD were calculated for experiments carried out in triplicate.

\section{Results}

Growth inhibition of TE-1 and ECA-109 cells by diphyllin. A standard CCK-8 assay was used to determine the prolif- eration (Fig. 1C and F) and cytotoxicity (Fig. 1A and B) of diphyllin in TE-1 and ECA-109 cells. After $48 \mathrm{~h}$ of incubation, the $\mathrm{IC}_{50}$ value of diphyllin was $0.2792 \mu \mathrm{M}$ in ECA-109 cells and $0.2058 \mu \mathrm{M}$ in TE- 1 cells (Fig. 1A and B).

Next, we assessed the effect of diphyllin on colony formation of ECA-109 and TE-1 cells. On day 11 post-treatment, diphyllin suppressed colony formation of the cells (Fig. 1G and H). 

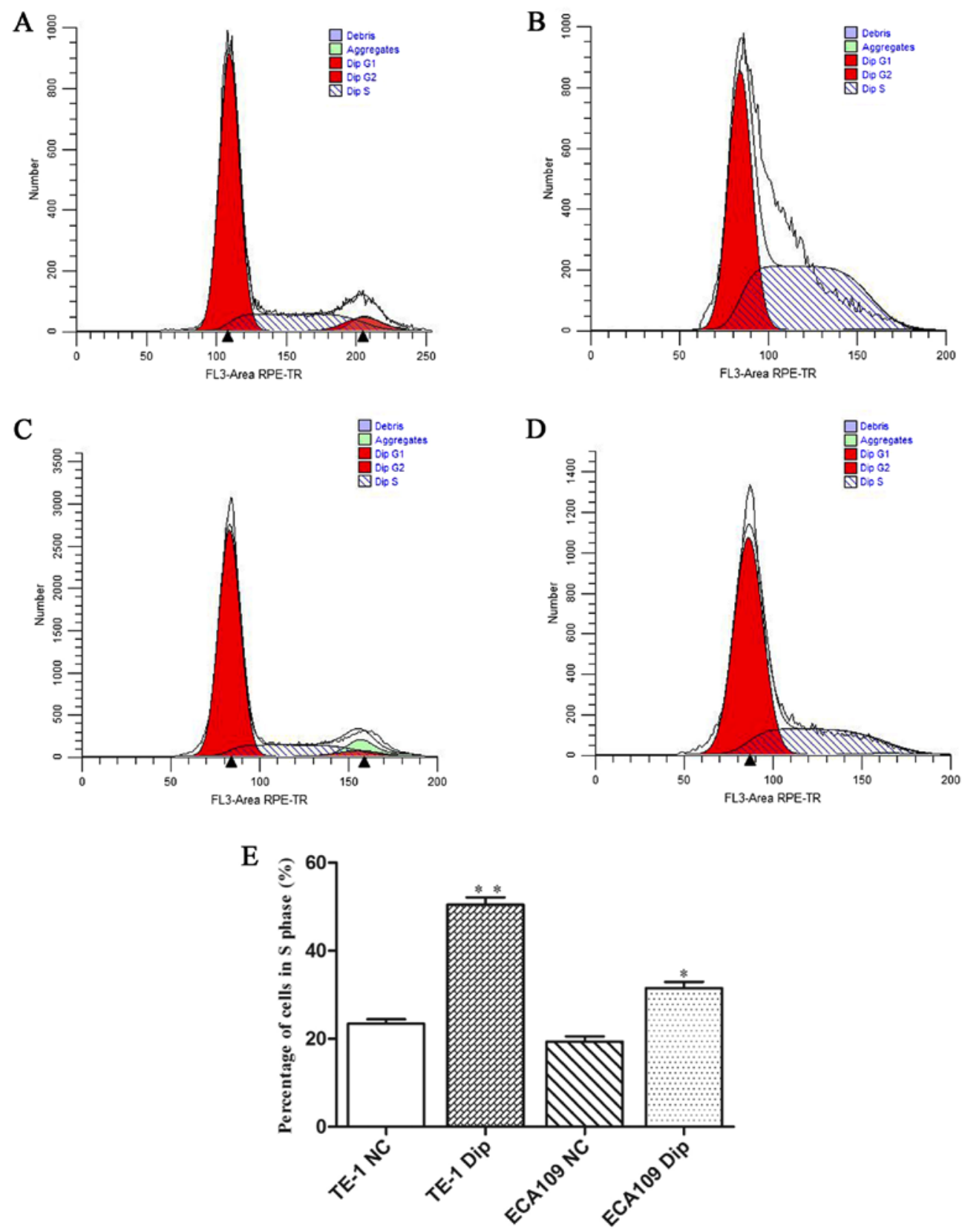

Figure 2. Flow cytometric assay was used to analyze cell cycle distribution. (A) The control group of TE-1 cells, no treatment. (B) Diphyllin group of TE-1 cells, treatment with diphyllin. (C) The control group of ECA-109 cells, no treatment. (D) Diphyllin group of TE-1 cells, treatment with diphyllin. (E) S phase cell percentage statistics for each group; ${ }^{*} \mathrm{P}<0.05,{ }^{* *} \mathrm{P}<0.01$.

Cell cycle distribution after diphyllin treatment. To determine the role of diphyllin in cell cycle distribution, flow cytometric analysis was performed. The results revealed that the diphyllin group exhibited significant $\mathrm{S}$ arrest compared with the normal group both in TE-1 and ECA-109 cells (Fig. 2).

Migration of TE-1 and ECA-109 cells treated with diphyllin. We investigated cell migration and found a decrease induced by diphyllin treatment in the Transwell assay. In addition, the scratch wound assay revealed inhibition of lateral cell migration (Fig. 3). These results demonstrated that diphyllin inhibited the migration of TE-1 and ECA-109 cells.

Effects of diphyllin on V-ATPase activity. We assessed the inhibitory effects of V-ATPase activity. ECA-109 and
TE-1 cells treated with diphyllin for $48 \mathrm{~h}$ were assessed for V-ATPase activity using a V-ATPase assay kit. After diphyllin treatment at various concentrations, the activity of V-ATPase was altered compared with the NC group. The activities of V-ATPase in $0.3 \mu \mathrm{M}$ diphyllin groups were significantly less than in the NC group $(\mathrm{P}<0.05)$. The activity of V-ATPase was also significantly decreased in cells treated with the same concentrations of bafilomycin for $48 \mathrm{~h}$ (Fig. 4).

Role of diphyllin in mTORC1/HIF-1 $\alpha / V E G F$ signaling. We further detected exocrine VEGF-C expression of cells treated with diphyllin. After $24 \mathrm{~h}$ of diphyllin treatment, VEGF-C levels were decreased in culture supernatants (Fig. 5A and B). Then, we detected the mRNA expression of VEGF-C, HIF- $1 \alpha$ and mTORC1 in TE-1 and ECA-109 cells treated with 


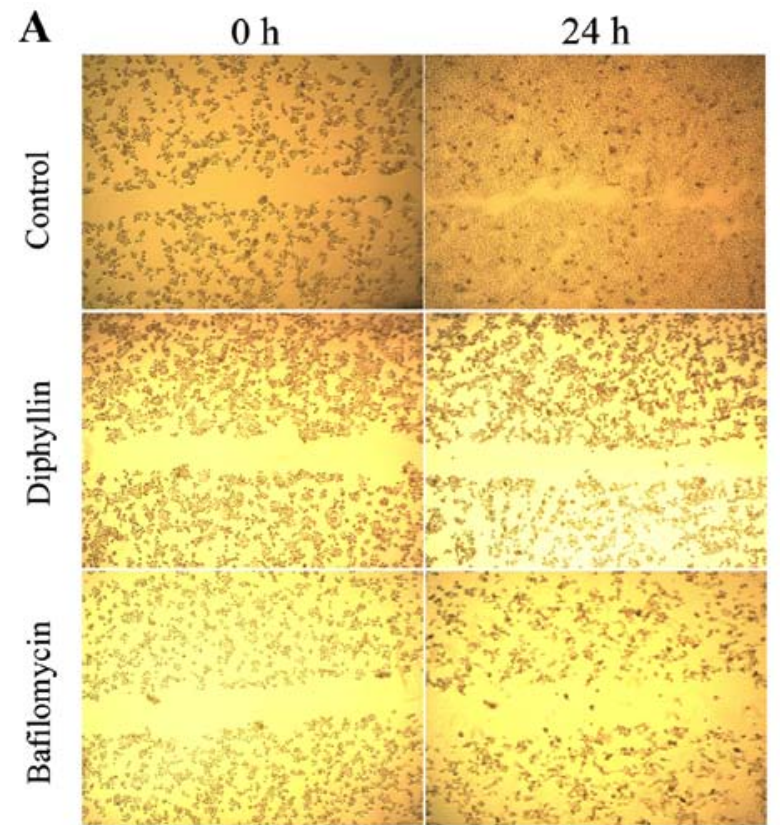

B

$0 \mathrm{~h}$

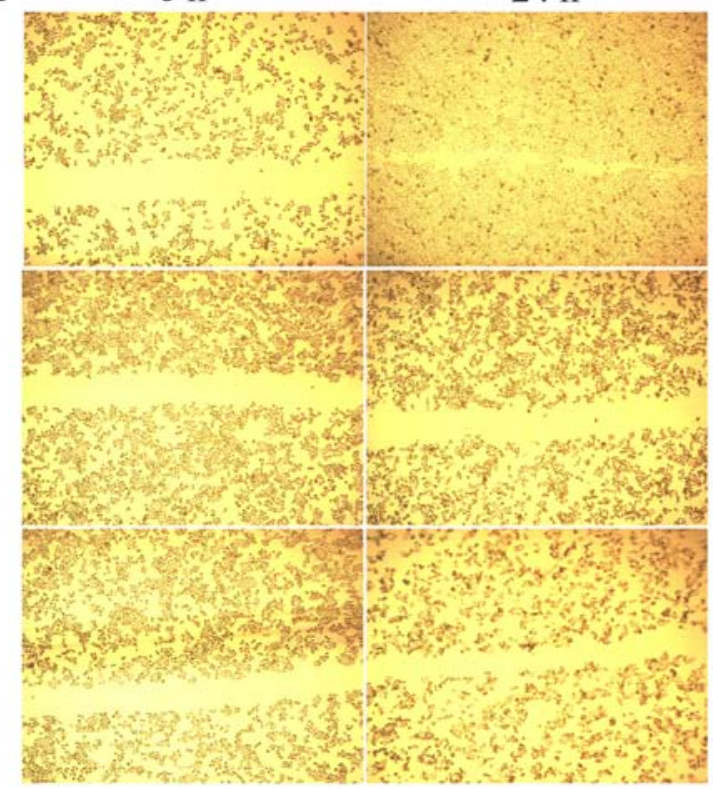

C

D
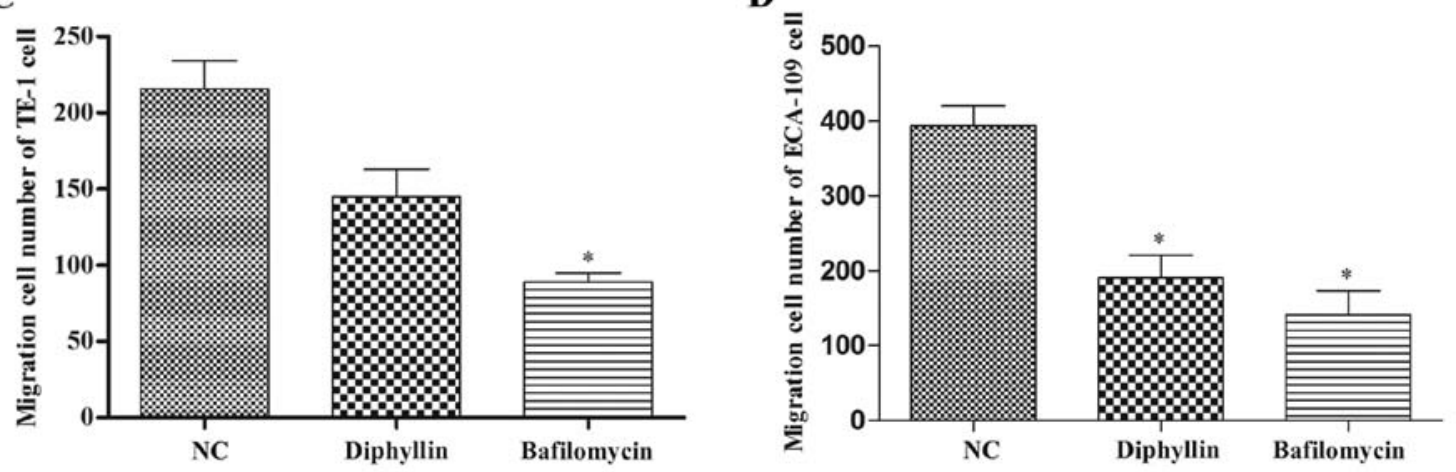

Figure 3. (A) Scratch wound assay of TE-1 cells. (B) Scratch wound assay of ECA-109 cells (magnification, $x 40)$. (C and D) Results of the Transwell assays (C, TE-1; and D, ECA-109 cells). The diphyllin and bafilomycin groups were compared with the negative control (NC) group; ${ }^{*} \mathrm{P}<0.05$

$\mathbf{A}$

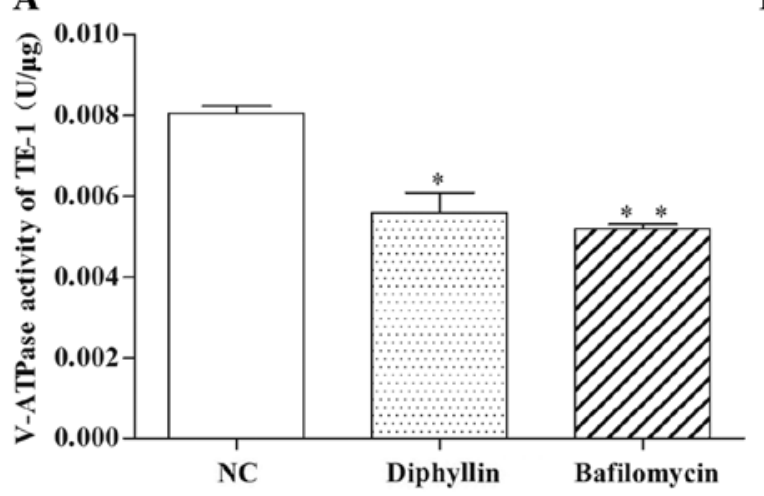

B

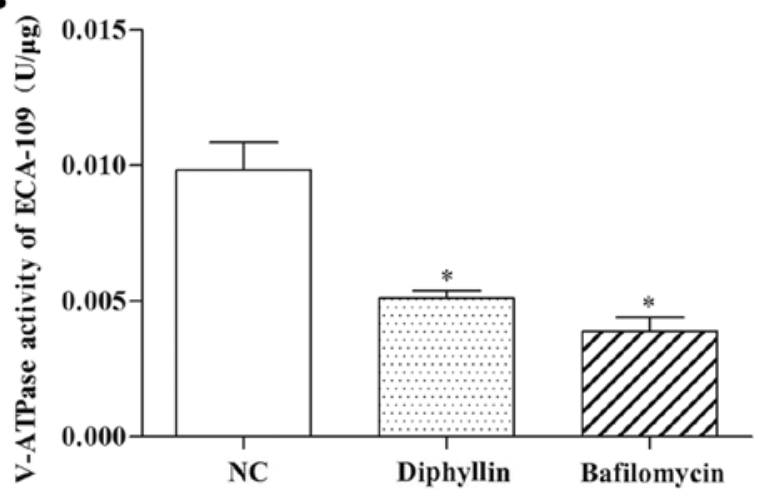

Figure 4. V-ATPase activities of (A) TE-1 and (B) ECA-109 cells were significantly decreased after treatment with diphyllin and bafilomycin. The diphyllin and bafilomycin groups were compared with the NC group; ${ }^{*} \mathrm{P}<0.05,{ }^{* *} \mathrm{P}<0.01$.

diphyllin or bafilomycin. The results revealed that diphyllin inhibited mTORC1, HIF-1 $\alpha$ and VEGF-C mRNA expression (Fig. 5C and D).

Proliferation of HUVECs treated with various culture supernatants. TE-1 and ECA-109 cells were treated for $24 \mathrm{~h}$ with diphyllin or bafilomycin, and their culture supernatants were used to treat HUVECs for $24 \mathrm{~h}$. The proliferation, migration and tube formation of HUVECs were detected. The results revealed that HUVEC proliferation, migration and tube formation ability were decreased significantly in the drug treatment groups compared with the control group. 
A

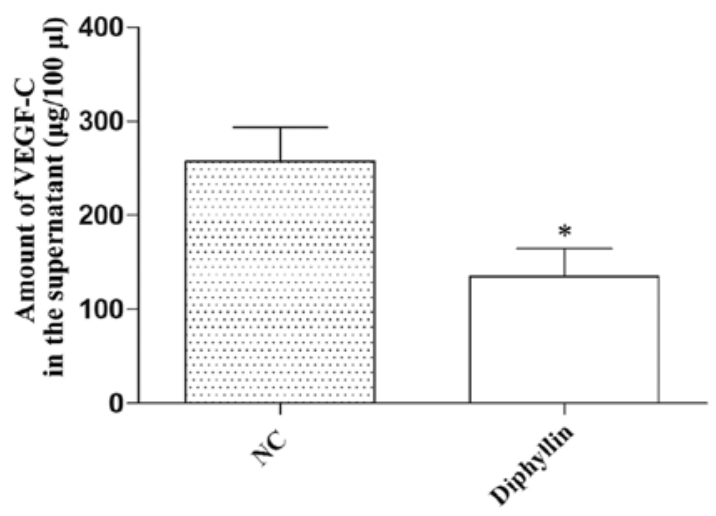

C

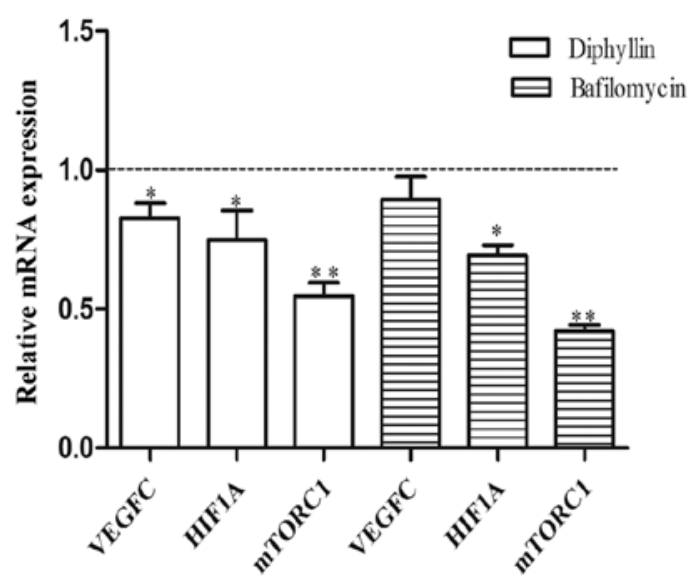

B

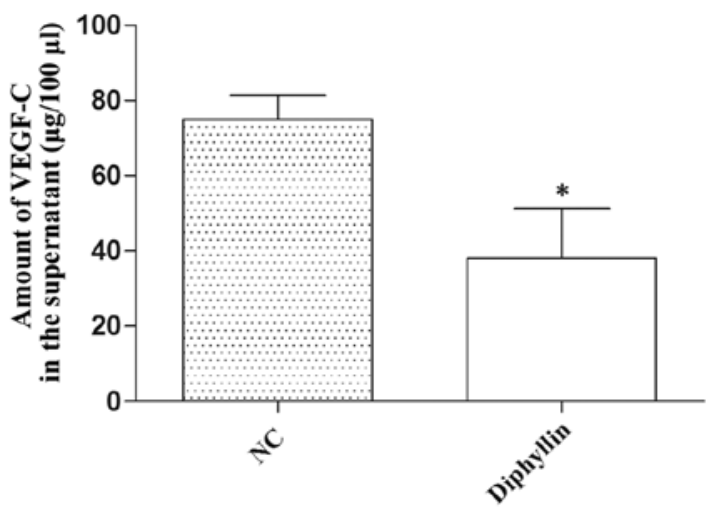

D

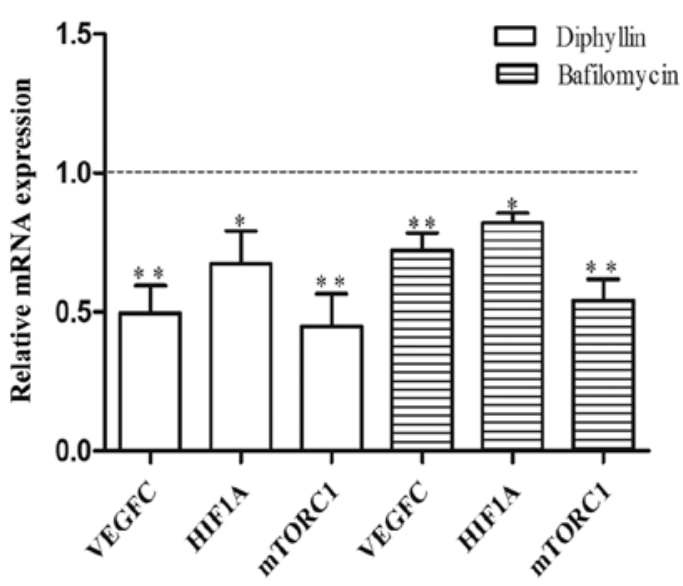

Figure 5. (A and B) Expression of VEGF-C in (A) TE-1 and (B) ECA-109 cells. (C and D) Relative mRNA expression. ${ }^{*} \mathrm{P}<0.05$, ${ }^{* *} \mathrm{P}<0.01$.

\section{Discussion}

Diphyllin is a natural component of traditional Chinese medicine with a naphthalene and one hydroxyl lignans (12-14), which promotes blood circulation and exerts a detoxicating detumescence effect. Recent studies have found that diphyllin also possesses an antitumor effect with few side-effects, but its exact mechanism of action is unclear. We treated esophageal TE-1 and ECA-109 cells with diphyllin and found inhibition of their proliferation and migration as well as induction of cell cycle arrest. These results revealed that diphyllin can be used as a tumor suppressor in esophageal cancer.

Regulation of lymphangiogenesis by VEGF signaling is an important mechanism of tumor metastasis $(6,7,13)$. When tumor cells proliferate to a certain extent, they secrete a number of lymphatic growth regulators of which the most important is VEGF. In the present study, we found that diphyllin inhibited VEGF expression in esophageal cancer cells, indicating that diphyllin inhibits metastasis through VEGF signaling, but the mechanism remains to be elucidated.

Recent studies revealed that mTOR regulates the secretion of VEGF in tumor cells by HIF-1 $\alpha$, thereby affecting the formation of new tumor vessels $(9,15,16)$. mTOR forms two protein complexes with different structures and functions (mTORC1 and mTORC2), and mTORC1 plays a role in regulation of HIF-1 $\alpha(15,16)$. Lysosomal $\mathrm{pH}$ value within tumor cells is significantly lower than in normal cells, which is conducive to increased cell autophagy and intracellular free amino acids, thereby activating mTORC 1 signaling, regulating HIF-1 $\alpha$, and promoting lymphangiogenesis and secretion of VEGF. Conversely, when the acidic environment of lysosomes within tumor cells is decreased, autophagy diminishes, intracellular free amino acid content decreases, and mTORC1 signaling is suppressed, thereby preventing tumor metastasis and accelerating lymphatic tumor cell death $(10,17)$. Studies have shown that maintenance of the acidity of mature lysosomes is closely related to V-ATPase $(5,6,23)$.

Recent studies indicate that diphyllin inhibits the activity of V-ATPase $(18,19)$, but there are no studies on esophageal cancer cells. V-ATPase expression in the cell membrane and membrane vesicles of tumor cells are significantly higher than that in normal tissue (3). $\mathrm{H}^{+}$is pumped out or into vesicles, causing extracellular vesicle and lysosomal acidification to maintain the acidic environment of tumor cells and vesicles, thereby limiting damage by $\mathrm{H}^{+}$and promoting proliferation, invasion, and metastasis (20-23). We determined that treatment of esophageal cancer cell lines TE-1 and ECA-109 with diphyllin led to a decrease in V-ATPase activity, inhibition of mTORC1, HIF-1 $\alpha$, and VEGF mRNA expression, and a decrease in secreted VEGF-C protein levels. Therefore, we hypothesized that diphyllin may inhibit metastasis through the V-ATPase/mTORC1/HIF-1 $\alpha /$ VEGF pathway. To test our hypothesis, we treated TE-1 and ECA-109 cells with diphyllin and used the culture supernatants to treat HUVECs. Thus, 
A

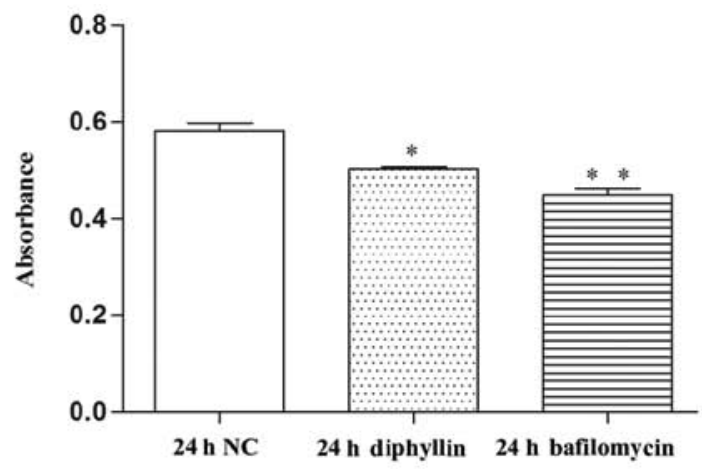

C

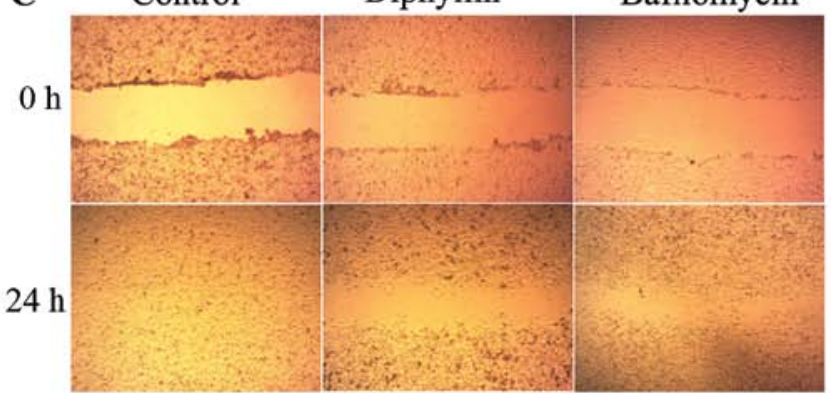

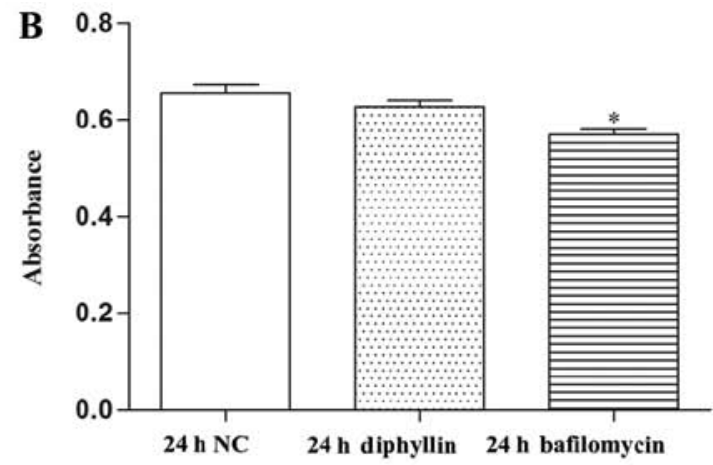

D

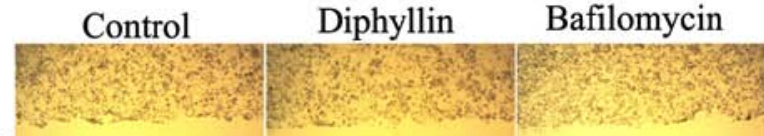

$0 \mathrm{~h}$

$24 \mathrm{~h}$

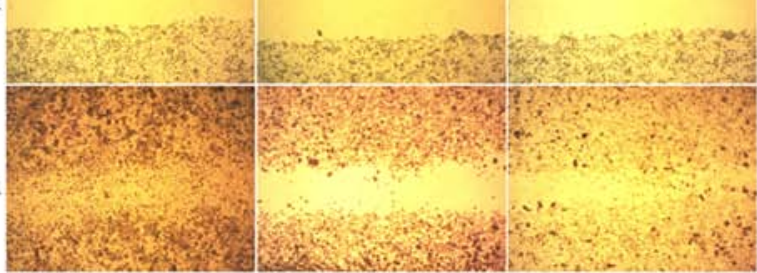

$\mathbf{E}$ Control Diphyllin Bafilomycin

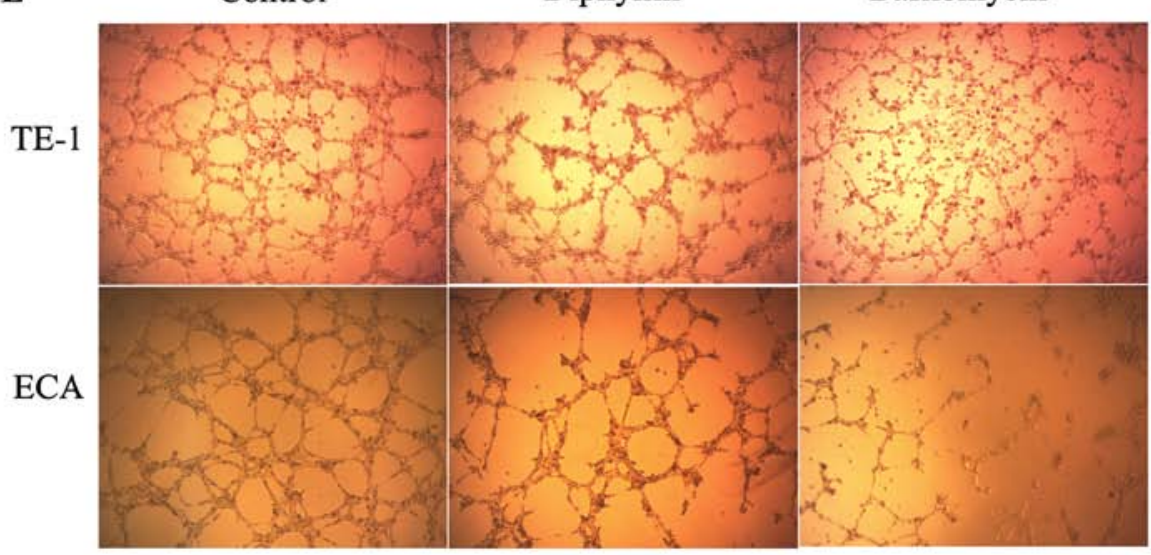

F

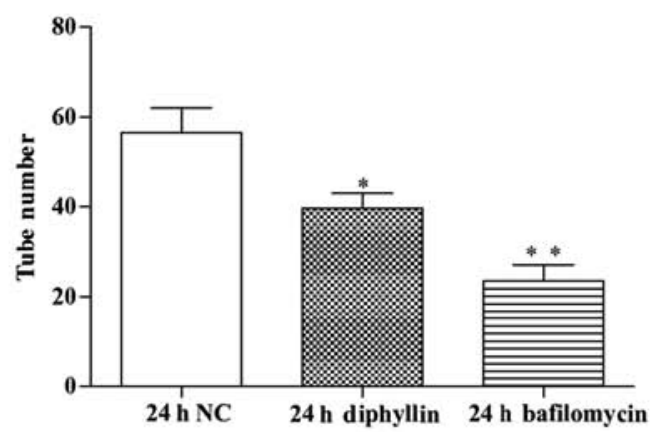

G

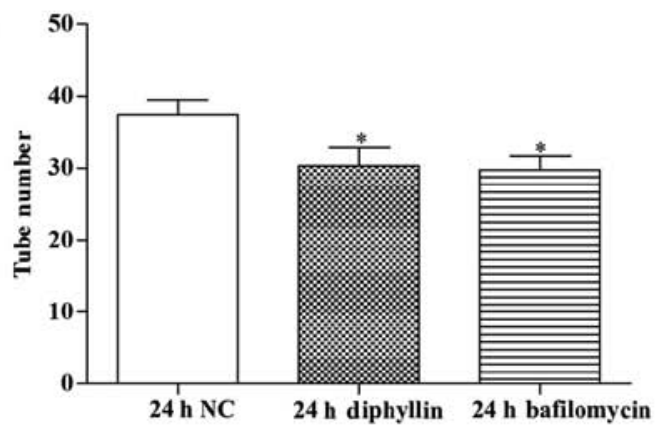

Figure 6. (A and B) Proliferation of HUVECs determined by CCK-8 assays (A, TE-1; and B, ECA-109 cells). (C and D) Scratch wound assay of HUVECs treated with various culture supernatants (C, TE-1; D, ECA-109 cells; magnification, x40). (E-G) Tube formation and the number of tube-like structures of HUVECs treated with various culture supernatants; ${ }^{*} \mathrm{P}<0.05,{ }^{* *} \mathrm{P}<0.01$.

diphyllin inhibited their proliferation, migration and tube formation.

In conclusion, diphyllin can be used as a tumor inhibitor to inhibit esophageal cancer cell proliferation and migration. As a new V-ATPase inhibitor, diphyllin may suppress metastasis of esophageal cancer. Its mechanism may be related to blocking the mTORC1/HIF-1 $\alpha /$ VEGF pathway inhibiting the formation of tumor vessels, but the specific mechanism remains to be elucidated.

\section{Acknowledgements}

The present study was supported by grants from the National Natural Science Foundation of China (General Program) 
(grant no. 31670857), the Jiangsu Provincial Natural Science Foundation of China (grant nos. BK20161152 and BK20171157), and the Open Research Fund of Jiangsu Key Laboratory of New Drug Research and Clinical Pharmacy (KF-XY201405).

\section{Competing interests}

The authors declare that they have no competing interests.

\section{References}

1. Zhang $\mathrm{X}$, Lin $\mathrm{Y}$ and Gillies RJ: Tumor $\mathrm{pH}$ and its measurement. J Nucl Med 51: 1167-1170, 2010.

2. Hernandez A, Serrano-Bueno G, Perez-Castineira JR and Serrano A: Intracellular proton pumps as targets in chemotherapy: V-ATPases and cancer. Curr Pharm Des 18: 1383-1394, 2012.

3. Shen W, Zou X, Chen M, Liu P, Shen Y, Huang S, Guo H and Zhang L: Effects of diphyllin as a novel V-ATPase inhibitor on gastric adenocarcinoma. Eur J Pharmacol 667: 330-338, 2011.

4. Siegel R, Naishadham D and Jemal A: Cancer statistics, 2013. CA Cancer J Clin 63: 11-30, 2013.

5. Gomes FG, Nedel F, Alves AM, Nör JE and Tarquinio SB: Tumor angiogenesis and lymphangiogenesis: Tumor/endothelial crosstalk and cellular/microenvironmental signaling mechanisms. Life Sci 92: 101-107, 2013.

6. Liu P, Chen W, Zhu H, Liu B, Song S, Shen W, Wang F, Tucker S, Zhong B and Wang D: Expression of VEGF-C correlates with a poor prognosis based on analysis of prognostic factors in 73 patients with esophageal squamous cell carcinomas. Jpn J Clin Oncol 39: 644-650, 2009

7. Liu P, Zhou J, Zhu H, Xie L, Wang F, Liu B, Shen W, Ye W, Xiang B, Zhu X, et al: VEGF-C promotes the development of esophageal cancer via regulating CNTN-1 expression. Cytokine 55: 8-17, 2011.

8. Shin J, Lee HJ, Jung DB, Jung JH, Lee HJ, Lee EO, Lee SG Shim BS, Choi SH, Ko SG, et al: Suppression of STAT3 and HIF-1 alpha mediates anti-angiogenic activity of betulinic acid in hypoxic PC-3 prostate cancer cells. PLoS One 6: e21492, 2011.

9. Elias AP and Dias S: Microenvironment changes (in $\mathrm{pH}$ ) affect VEGF alternative splicing. Cancer Microenviron 1: 131-139, 2008.

10. Kraut JA and Madias NE: Metabolic acidosis: Pathophysiology, diagnosis and management. Nat Rev Nephrol 6: 274-285, 2010.
11. Le Tourneau C, Faivre S, Serova M and Raymond E: mTORC1 inhibitors: Is temsirolimus in renal cancer telling us how they really work? Br J Cancer 99: 1197-1203, 2008.

12. Liu P, Chen H, Han L, Zhou X and Shen W: Expression and role of V1A subunit of V-ATPase in gastric cancer cells. Int J Clin Oncol 20: 725-735, 2015.

13. Das S, Ladell DS, Podgrabinska S, Ponomarev V, Nagi C, Fallon JT and Skobe M: Vascular endothelial growth factor-C induces lymphangitic carcinomatosis, an extremely aggressive form of lung metastases. Cancer Res 70: 1814-1824, 2010.

14. Wang Y, Xia C, Zhang W and Zhao Y: Synthesis and biological evaluation of novel lignan glycosides as anticancer agents. Chem Biol Drug Des 88: 562-567, 2016.

15. Efeyan A, Zoncu R, Sabatini DM, Wang S and Sancak Y: Amino acids and mTORC1: From lysosomes to disease. Trends Mol Med 18: 524-533, 2012.

16. Balgi AD, Diering GH, Donohue E, Lam KK, Fonseca BD, Zimmerman $C$, Numata $M$ and Roberge $M$ : Regulation of mTORC1 signaling by pH. PLoS One 6: e21549, 2011.

17. Naito T, Kuma A and Mizushima N: Differential contribution of insulin and amino acids to the mTORC1-autophagy pathway in the liver and muscle. J Biol Chem 288: 21074-21081, 2013.

18. Sørensen MG, Henriksen K, Neutzsky-Wulff AV, Dziegiel MH and Karsdal MA: Diphyllin, a novel and naturally potent V-ATPase inhibitor, abrogates acidification of the osteoclastic resorption lacunae and bone resorption. J Bone Miner Res 22: 1640-1648, 2007.

19. Zhao Y, Ni C, Zhang Y and Zhu L: Synthesis and bioevaluation of diphyllin glycosides as novel anticancer agents. Arch Pharm 345: 622-628, 2012.

20. Zoncu R, Bar-Peled L, Efeyan A, Wang S, Sancak Y and Sabatini DM: mTORC1 senses lysosomal amino acids through an inside-out mechanism that requires the vacuolar $\mathrm{H}^{+}$-ATPase. Science 334: 678-683, 2011

21. Zhou M, Morgner N, Barrera NP, Politis A, Isaacson SC, MatakVinković D, Murata T, Bernal RA, Stock D and Robinson CV: Mass spectrometry of intact V-type ATPases reveals bound lipids and the effects of nucleotide binding. Science 334: 380-385, 2011.

22. Shen W, Zou X, Chen M, Shen Y, Huang S, Guo H, Zhang L and Liu P: Effect of pantoprazole on human gastric adenocarcinoma SGC7901 cells through regulation of phospho-LRP6 expression in Wnt/ $\beta$-catenin signaling. Oncol Rep 30: 851-855, 2013.

23. Zhao Y, Lu Y, Ma J and Zhu L: Synthesis and evaluation of cleistanthin A derivatives as potent vacuolar $\mathrm{H}^{+}$-ATPase inhibitors. Chem Biol Drug Des 86: 691-696, 2015.

This work is licensed under a Creative Commons Attribution-NonCommercial-NoDerivatives 4.0 International (CC BY-NC-ND 4.0) License. 